\title{
bioRXiv
}

\section{EXOSOME RELEASE PROMOTES INFLAMMATORY RESOLUTION IN ACTIVATED AND AGED MICROGLIA}

\author{
Joe C. Udeochu1-3, Cesar Sanchez-Diaz1, Alvan Cai1, Anna Jovicic4, Saul A. Villeda1-3,5,\#
}

Proper regulation of inflammatory responses is critical for effective function of microglia, in both physiological and disease states. While the mechanisms that drive microglia activation are well characterized, the pathways leading to inflammatory resolution and immune homeostasis have yet to be fully elucidated. Using RNA interference, pharmacological inhibition and genetic knockout mouse model approaches, we show that exosome release promotes immune homeostasis in activated and aged microglia. We demonstrate that induction of anti-inflammatory pathways enhances release of exosomes containing immune proteins and microRNAs. Functionally, inhibition of exosome release alters trafficking of exosome cargo, such as miR-155, in activated microglia resulting in increased cellular retention of these cargo molecules. Concordantly, we identify increased miR-155 activity leading to sustained activation of pro-inflammatory pathways as a potential mechanism underlying impaired inflammatory resolution due to inhibition of microglia exosome release. Similarly, inhibition of augmented exosome release in aged microglia exacerbates inflammatory activation, demonstrating conservation of the immune modulatory effects of exosome release in microglia. Taken together, our study identifies exosomes as novel components of an anti-inflammatory mechanism utilized by activated and aged microglia to restore immune homeostasis.

\section{INTRODUCTION}

As the tissue macrophages of the brain, microglia activity is strongly tailored to meet the specialized needs of their environment (Kettenmann et al., 2011; Nimmerjahn et al., 2005). Increased brain inflammatory milieu during aging and disease, however, drives transformation of microglia from a homeostatic to an activated state (Hickman et al., 2013; Prinz et al., 2011; Udeochu et al., 2016). Numerous factors including increased cytokine production, cellular senescence, and loss of neuronal 'off' signals have been implicated in driving microglia inflammatory transformation (Wong, 2013; Ye and Johnson, 1999). However, much still remains to be learned about mechanisms that counterbalance the effects of inflammation to facilitate immune homeostasis in activated aged and diseased microglia.

The endolysosomal network is central for maintenance of cell homeostasis, through trafficking, signaling, and degradation of both endocytic and biosynthetic cargoes (Klumperman and Raposo, 2014). Exosomes, a subset of endosomal-derived extracellular vesicles, have emerged as critical regulators of various aspects of peripheral immune cell function including inflammatory activation and suppression (Lindenbergh and Stoorvogel, 2018; Robbins and Morelli, 2014). Moreover, mutations in regulator of exosome release, Rab27a, cause Griscelli syndrome, which is characterized by peripheral macrophage hyperactivation and cellular infiltration of the brain (Menasche et al., 2000; Sarper et al., 2002). Recent studies have demonstrated microglia uptake of exosomes produced by neurons and other glia (Fitzner et al., 2011; Fruhbeis et al., 2013), and regulation of neuropeptide catabolism by microglia exosomes (Potolicchio et al., 2005). Exosome involvement in microglia inflammation is, however, unclear. To address this, we utilized molecular and genetic techniques to assess exosomal regulation of inflammation in cultured, adult brain and aged microglia. Specifically, we studied the effects of microglia activation on exosome biogenesis, manipulated exosome biogenesis experimentally, and determined a molecular mechanism mediating exosomal regulation of immune homeostasis in activated microglia.

\section{RESULTS}

Microglia produce canonical exosomes that contain cell type-specific factors.

To begin our study, we characterized exosome production in primary microglia and BV2 immortalized microglia cell line cultured under serum free conditions. Secreted exosomes were isolated from media by standard

1. Department of Anatomy, University of California San Francisco, San Francisco, California 94143, USA

2. The Eli and Edythe Broad Center for Regeneration Medicine and Stem Cell Research, San Francisco, California 94143, USA

3. Biomedical Sciences Graduate Program, University of California San Francisco, San Francisco, California 94143, USA

4. Department of Genetics, Stanford University, Stanford, California 94305, USA

5. Department of Physical Therapy and Rehabilitation Science, University of California San Francisco, San Francisco, California 94143, USA Corresponding Author: Dr. Saul Villeda, PhD

University of California San Francisco, Department of Anatomy

513 Parnassus Ave, Box 0452, San Francisco, CA 94143 


\section{bioRxiv}

filtration and differential ultracentrifugation (Thery et al., 2006). Analysis of physical characteristics and protein content revealed enrichment of exosomes in our preparations. Specifically, scanning electron microscopy and nanoparticle tracking analysis (NTA) showed that microglia exosomes exhibit stereotypical donut-shaped morphology and were on average 30$120 \mathrm{~nm}$ in size (Figures $1 \mathrm{~A}$ and $\mathrm{B}$ ). Detection of tetraspanin proteins, CD9 and CD63, and endolysosomal protein, LAMP2, further confirmed exosome enrichment (Figure 1C). We next tested the involvement of ceramide synthase pathway and Rab GTPases in microglia exosome biogenesis by inhibiting neutral sphingomyelinase 2 (nSMase2) and Rab27a, respectively (Ostrowski et al., 2010; Trajkovic et al., 2008). Transduction of BV2 microglia with lentiviral particles expressing Rab27a targeting (KD) short hairpins RNAs (shRNAs) led to efficient Rab27a knockdown (Figure 1D) and reduced exosome secretion into media compared to cells expressing scrambled (scr) con-

\section{ARTICLE PREPRINT}

A

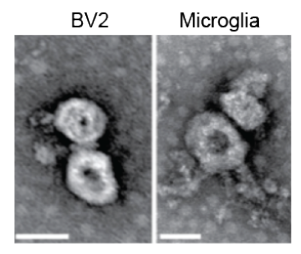

D

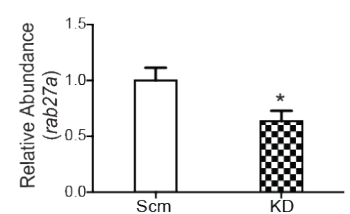

G

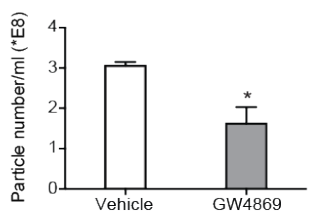

B

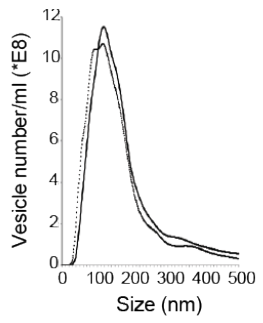

E

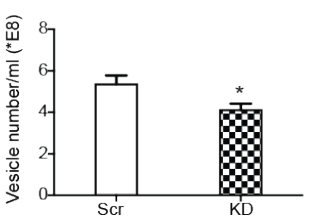

C

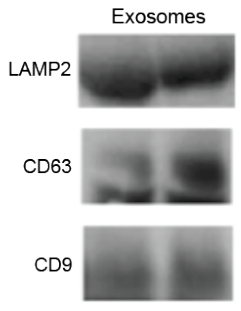

$\mathbf{F}$

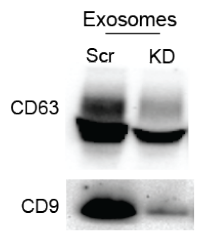

H

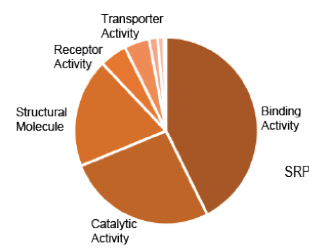

I

To elucidate the signaling potential of microglial exosomes, we characterized the protein and miRNA contents of exosomes produced by BV2 microglia. Mass spectrometry and miRNA array analyses revealed that microglia exosomes contain heterogeneous cargo, as has been reported for exosomes from other cell types (Kowal et al., 2016; Squadrito et al., 2014) (Figure 1H, Supplemental Table 1 and 2). We identified canonical exosome content such ALIX and MFGE8 proteins, as well as cargo unique to microglia and myeloid cells like mannose receptor, macrophage inhibitory protein, miR-146a, and miR-150 (Supplemental Table 1 and 2). Gene ontology analysis revealed that microglia exosomes were enriched in proteins involved in biological processes such as viral gene expression, protein trafficking, nucleic acid catabolism and degranulation (Figure 1I). The diversity of biological pathways represent-

trol sequences (Figures $1 \mathrm{E}$ and $1 \mathrm{~F}$ ). Similarly, treatment with the neutral sphingomyelinase inhibitor, GW4869, reduced microglia exosome production (Figure 1G). 
A
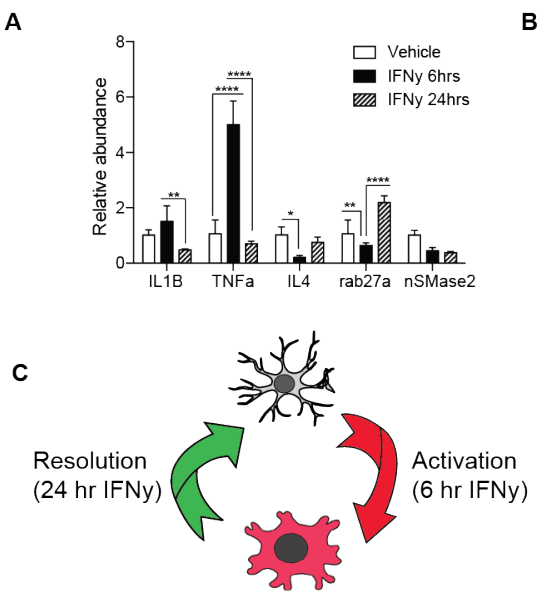

E

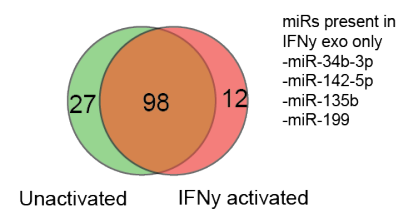

B

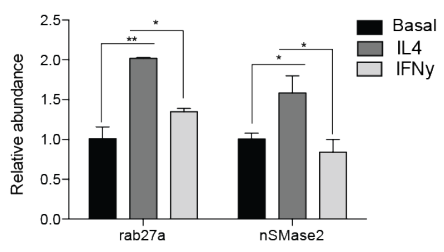

D

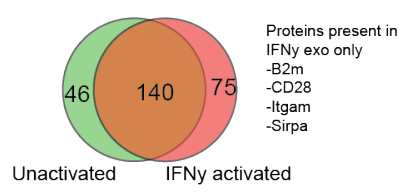

$\mathbf{F}$

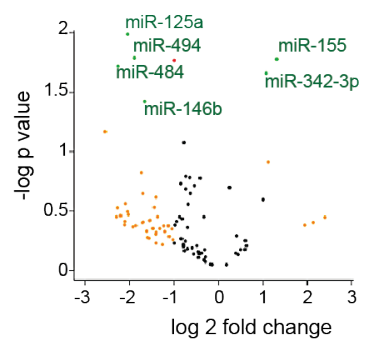

Figure 2. Inflammation regulates exosome production and cargo in microglia.

(A) Quantitative real-time PCR results of interferon gamma (IFN $\psi$ ) stimulated primary microglia showing expression of inflammatory cytokines (IL1 $\beta, T N F \alpha$, and IL4) and exosome regulatory genes (nSMase2 and rab27a). (B) Acute interleukin-4 stimulation significantly upregulates rab27a in BV2 microglia. (C) Schematic demonstrating model for IFN $\gamma$-induced inflammatory activation and resolution in microglia. (D-E) Comparison of protein (D) and microRNA (E) contents of exosomes derived from activated and unactivated microglia during resolution of IFN $\gamma$ inflammation. (F) Volcano plot showing IFN $\gamma$ induced enrichment of immune related miRNAs, miR-155 and miR-342-3p, in IFN exosomes compared unactivated exomes (yellow $=2$ fold change, red $=p<0.05$, green $=2$ fold change and $p<$ 0.05). All data represented as mean $\pm \mathrm{SEM} ;{ }^{*} \mathrm{P}<0.05 ;{ }^{* *} \mathrm{P}<0.01 ;{ }^{* * *} \mathrm{P}<0.0001$, one-way ANOVA $(\mathrm{A}), \mathrm{t}$-test $(\mathrm{B}, \mathrm{F})$.

ed in microglia exosome cargo suggested a potential role in regulating cellular responses and function.

\section{Microglia activation leads to recruitment of pro-inflammatory molecules into exosomes.}

To explore exosome involvement in microglia inflammatory response, we next assessed the effects of activation on the expression of exosome regulatory genes, rab27a and nSMase2. Quantitative real time PCR analyses of microglia stimulated with pro-inflammatory agents - interferon gamma (IFN $\gamma$ ), lipopolysaccharide (LPS), or polyribocytidilic acid (poly $\mathrm{I}: \mathrm{C})$, revealed activation induced changes in Rab27a and nSMase2 expression (Figure 2A and S1). Overall, pro-inflammatory activation suppressed microglia nSMase2 expression. On the other hand, Rab27a expression was more dynamically regulated in activated microglia. Strikingly, we note that kinetics of Rab27a expression mirrored interleukin 4 (IL-4) under all three activation paradigms. Consequently, we tested the possibility that IL-4 might coordinate Rab27a expression and show that recombinant IL-4 stimulation significantly increases mi- croglia Rab27a (Figure 2B). These findings show that exosome regulatory genes are responsive to microglia activation, and suggest an anti-inflammatory function for exosome release in acutely activated microglia.

Further inspection of cytokine gene expression kinetics revealed that the 6 and 24 hour IFN $\gamma$ stimulation timepoints represent two distinct phases of inflammation - activation and resolution phases, respectively (Figure 2C). Given that Rab27a is strongly upregulated during resolution and coincides with the repression of pro-inflammatory IL $1 \mathrm{~b}$ and TNFa, we next compared the protein and miRNA content of exosomes released by resolving and unstimulated BV2 microglia for differences in immune signatures. While exosomes from resolving and unstimulated microglia shared many proteins and miRNAs, we observed some activation induced differences in cargo (Figure 2F-2H). There was selective or increased recruitment of cargo implicated in immune cell activation, such as CD28, beta-2-microglobulin, miR-142-5p, and miR-155, (Ponomarev et al., 2013) into exosomes released by resolving microglia. This suggests that exosome cargo sorting in microglia is dynamic and partly influenced by microglia activation state. 


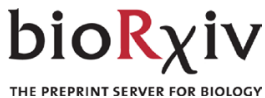

A

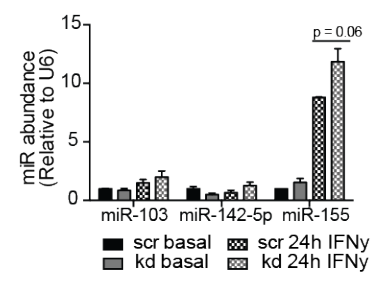

C

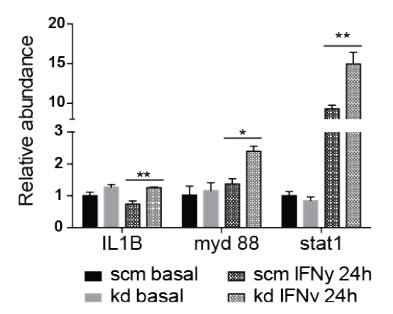

E

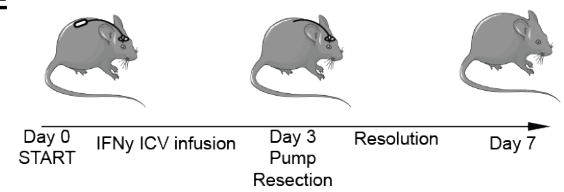

G

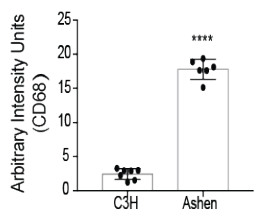

B

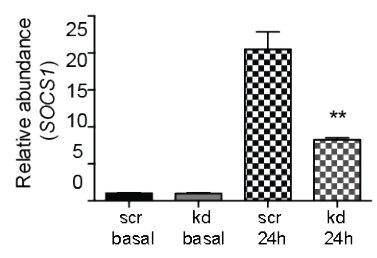

D

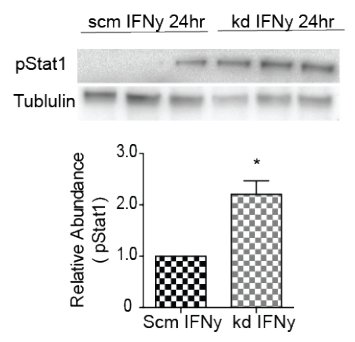

$\mathbf{F}$

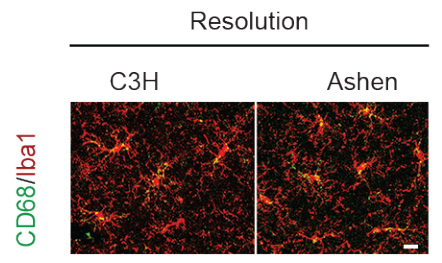

H
Figure 3. Inhibition of exosome productions alters miR-155 dynamics and impairs resolution of acute inflammation in cultured and adult brain microglia. (A) Quantitative PCR analyses of IFN $\gamma$ exosome enriched miRNAs, showing increased cellular miR-155 levels in activated rab27a KD compared to scm BV2. (B) Corresponding decrease in expression of miR-155 target, suppressor of cytokinen signaling (SOCS1), in activated rab27a KD BV2. (C) Increased transcript levels of pro-inflammatory mediators IL $1 \beta$, myd88 and Stat1 in rab27a KD compared to $\mathrm{scm}$ BV2 microglia. (D) Sustained STAT1 phosphorylation in rab27a KD compared scm BV2 microglia 24 hours post IFN $\gamma$ activation. (E) Intracerebroventricular (ICV) osmotic infusion paradigm utilized to assess IFN $\gamma$ induced inflammatory resolution in 3 month old wild type $(\mathrm{C} 3 \mathrm{H})$ and rab27a mutant (Ashen) adult brain microglia. (F) Representative confocal images showing higher CD68 immunoreactivity in Iba1+ microglia in Ashen compared to $\mathrm{C} 3 \mathrm{H}$ brains, quantified in (G). (H) Quantitative PCR analyses showing increased Stat1 and IL6 transcript levels in Ashen compared to $\mathrm{C} 3 \mathrm{H}$ brain homogenates, indicating impaired resolution of IFN $\gamma$ inflammation. Data represented as mean \pm SEM; ${ }^{*} \mathrm{P}<0.05$; ${ }^{*} \mathrm{P}<0.01$; unpaired t-test $(A-D, G-H)$. Scale bar $=20 u m$.

\section{Exosome release is necessary for efficient reso- lution of inflammation in microglia.}

Given the observed enrichment of immune related miRNAs in IFN $\gamma$ exosomes, we next assessed the consequences of inhibiting exosome biogenesis on the trafficking of these miRNAs. While abrogation of Rab27a had no effect on basal levels of miR-103 and miR-155, we observed increased cellular accumulation of miR-155 in resolving Rab27a KD BV2 compared to scr controls (Figure 3A). Similarly, GW4869 treatment increased cellular accumulation of miR-142$5 p$ and miR-155 after IFN $\gamma$ stimulation (Figure S2A). miR-155 activity amplifies interferon induced JAK/ STAT signaling in part by repressing inhibitors of immune response such as the suppressor of cytokine signaling (SOCS1) (Wang et al., 2010). Quantitative PCR analyses revealed significantly lower SOCS1 expression in Rab27a KD and GW4869 treated BV2 compared to respective controls, indicating increased miR-155 activity (Figures 3B and S2B). To determine if these observed changes in exosomal miR-155 trafficking resulted in broad inflammatory dysregulation in activated microglia, we measured the expression of various pro-inflammatory mediators of IFN $\gamma$ signaling. Indeed, resolving Rab27a KD microglia expressed significantly higher levels of IL $1 \beta$, myd88, and Stat 1 compared to scr controls (Figure 3C). GW4869 treatment resulted in similar but milder effects on microglia activation, in line with data in Figure 2 showing more prominent induction of Rab27a compared to nSmase2 during resolution of IFN $\gamma$ inflammation (Figure S2C). Concordantly, resolving Rab27a KD and GW4869-treated microglia exhibited increased Stat1 phosphorylation levels compared to controls, indicating sustained activation of JAK-STAT signaling (Figure 3D and S2D). These findings highlight a novel role of exosomes in microglia immune homeostasis, wherein increasing exosome release promotes clearance of immune miRNAs like miR-155 to allow inflammatory resolution. Next, we examined whether inhibiting exosome release in the adult brain would also impairs resolution 


\section{bioRxiv}

of microglia inflammation after acute IFN $\gamma$ stimulation. We compared microglia activation in wild type $\mathrm{C} 3 \mathrm{H}$ mice and Ashen mice, which harbor a spontaneously generated point mutation in Rab27a that results in a hypomorphic allele. Under baseline conditions, no differences in microglia activation were observed between young ( 3 months) Ashen mice and $\mathrm{C} 3 \mathrm{H}$ controls, as assessed by immunofluorescence analysis of the microglia-specific calcium binding adaptor protein, Iba1, and the activation marker, CD68, respectively (Figures S3A and B). To model resolution of IFN $\gamma$-mediated inflammation, we perfomed a modified intracerebroventricular (ICV) infusion paradigm on 3-month old $\mathrm{C} 3 \mathrm{H}$ and Ashen brains. Mice received ICV IFN $\gamma$ infusion, after which osmotic pumps were surgically resected and mice allowed four days of recovery (Figure 3E). Immunofluorescent analysis post recovery revealed low levels of CD68 immunoreactivity in $\mathrm{C} 3 \mathrm{H}$ wild type microglia indicating effective inflammatory resolution of IFN $\gamma$-induced inflammation. Ashen microglia, however, maintained significantly higher CD68 expression indicating impaired resolution (Figure 3F and 3G). In addition, higher Stat1 and IL6 levels show sustained IFN $\gamma$ activation in the resolving Ashen compared to $\mathrm{C} 3 \mathrm{H}$ brains (Figure $3 \mathrm{H}$ ). We also analyzed microglia activation immediately after three days IFN $\gamma$ infusion (Figure S3C) and observed no differences in CD68 expression between Ashen and $\mathrm{C} 3 \mathrm{H}$ microglia (Figures. S3D and $\mathrm{E}$ ). Thus, the differences reported here between Ashen and $\mathrm{C} 3 \mathrm{H}$ microglia during inflammatory resolution are unlikely due to differential IFN $\gamma$ activation (Figures. S3D and E). Our findings demonstrate exosome release is required for efficient inflammatory resolution in acutely activated young adult brain microglia.

\section{Augmented exosome release modulates inflam- matory activation in aged microglia.}

Microglia aging is accompanied by increased production of inflammatory molecules resulting in a chronic state of activation that drives morphological and functional changes in microglia (Mosher and Wyss-Coray, 2014; Sierra et al., 2007). Upregulation of interferon signaling and other antiviral response pathways has recently been identified as one of the transcriptional signatures associated with activation in aged microglia (Baruch et al., 2014; Grabert et al., 2016). Considering our findings implicating exosomes in interferon responses in acutely activated microglia, we examined the potential involvement of exosomes in aging-associated chronic microglia
ARTICLE PREPRINT

activation. We first compared the abundance of interstitial exosomes between young and aged brains, using a previously published protocol (Perez-Gonzalez et al., 2012). Interstitial exosomes were higher in aged compared to young brains, based on increased CD9 and CD63 immunolabeling and nanoparticle tracking analyses (Figures 4A-4C). To directly test the effects of aging on microglia exosome production, we utilized qPCR analyses of exosome regulatory genes in acutely isolated young and aged microglia. Higher transcript levels of IL1 $\beta$ and IL 10 in aged microglia confirmed the activated state of aged microglia compared to young (Figure 4D). Interestingly, we also observed increased rab27a expression in aged microglia, indicating that microglia activation in the aged brain is accompanied by increased exosome release (Figure 4D). Furthermore, immunofluorescence analysis revealed higher CD63 levels in aged compared to young microglia (Figures 4E and 4F). These data demonstrate age-dependent alterations in microglia exosome production and suggest that the increase of exosome abundance in the aged brain derives, at least in part, from increased exosome release by aged microglia.

Lastly, we asked if augmented exosome production is involved in the regulation of inflammation in aged microglia. We performed stereotaxic injections of exosome inhibitor, GW4869, or vehicle into contralateral hippocampi of young (3 months) and aged (18 months) mice. Microglia activation was assessed one week post-injection by CD68 immunofluorescence analyses. No differences in CD68 expression were detected between GW4869 and vehicle injected conditions in the young brain (Figures $4 \mathrm{G}$ and $4 \mathrm{H}$ ). In the aged brain, however, microglia CD68 expression was significantly higher in GW4869- compared to vehicle-injected hippocampi (Figures $4 \mathrm{I}$ and $4 \mathrm{~J}$ ). These data show that augmented exosome release is an important anti-inflammatory mechanism utilized by microglia to modulate aging-associated chronic inflammatory activation.

\section{DISCUSSION}

Microglia transform to an activated state in response to inflammatory challenge and changes in brain homeostasis during aging and disease. There is increasing evidence that sustained microglia activation can contribute to brain pathology and dysfunction under these conditions. A better understanding of microglia inflammatory processes can inform ongoing efforts to preserve and restore function in aged and diseased brains. Our study focuses not on drivers of microglia activation, but on mechanisms of inflammatory resolution that al- 
A

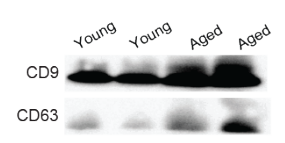

C

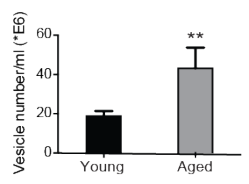

E

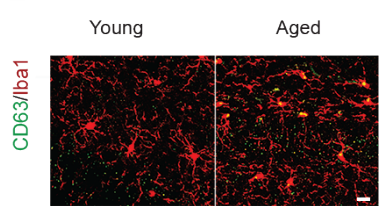

G

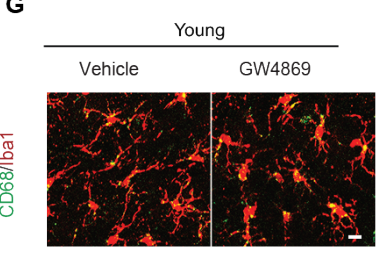

I

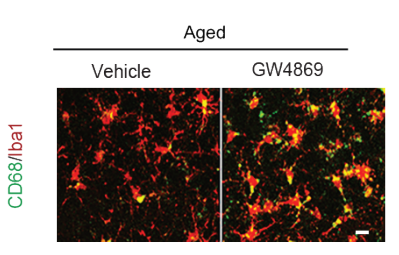

B

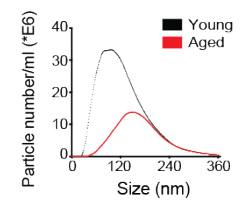

D

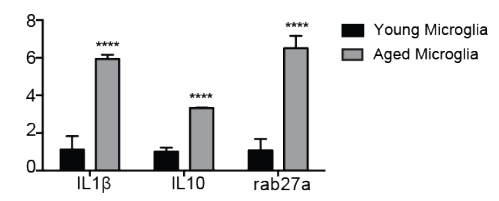

$\mathbf{F}$
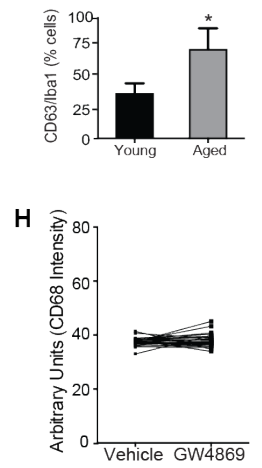

$\mathbf{J}$

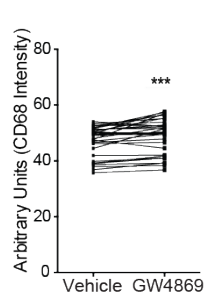

Figure 4. Increased exosome production modulates inflammatory activation in aged microglia

(A) Representative western blot probes for tetraspanin proteins, CD9 and CD63, in exosomes isolated from young (3 months) and aged (18 months) hemibrains. (B) Representative nanoparticle tracking traces of young and aged brain exosomes. (C) Nanoparticle tracking analyses showing significantly higher abundance of interstitial exosomes in aged compared to young brains. (D) Quantitative PCR of acutely isolated young and aged exosomes, showing upregulated expression of inflammatory (II1B, II10) and exosome regulatory (rab27a) genes. (E) Representative immunofluorescent images of microglia (Iba1) colabeled with exosome marker (CD63). (F) Quantification of hippocampal microglia showing aging-associated increase in microglial CD63. (G,I) Representative immunofluorescent images of vehicle and GW4869 injected hemibrains stained for microglia marker, Iba1, and activation marker, Cd68. (H) No difference in CD68 intensity in young vehicle and GW4869 hemibrains. (J) GW4869 treatment results in significantly higher CD68 expression compared to vehicle treated hemibrains in aged mice. Data represented as mean $\pm \mathrm{SEM}$; ${ }^{*} \mathrm{P}<0.05$; ${ }^{* *} \mathrm{P}<0.01$; ${ }^{* * * *} \mathrm{P}<0.0001$; t-test $(\mathrm{C}, \mathrm{D}, \mathrm{F}, \mathrm{H}, \mathrm{J})$. Scale bar $=20 \mu \mathrm{m}$.

low microglia to counterbalance activation signals. We identify the upregulation of exosome release as an important effector mechanism that promotes immune homeostasis in acutely activated and aged microglia. We show that microglia upregulate Rab27a-dependent exosome release during resolution of interferon-induced activation, a process that is partly mediated by anti-inflammatory cytokine signaling. These findings are in line with results showing increased exosome release in interleukin-4 treated bone marrow derived macrophages (Squadrito et al., 2014). Additionally, exosomes released by resolving microglia contain higher levels of immune proteins and miRNAs involved in toll-like and interferon signaling, such as miR-155. miR-155 has been implicated in driving pro-inflammatory transcriptional signatures in macrophages and in aberrant microglia inflammatory activation in disease conditions (Butovsky et al., 2015; Jablonski et al., 2016). Our experiments show that exosome release is critical for regulating cellular levels and activity of miR-155 in resolving microglia. Reduction of exosome release leads to miR-155 accumulation in resolving microglia, and as result sustained activation of STAT1 signaling and impaired inflammatory resolution. Previous studies showing that multivesicular bodies (MVB), the site of exosome formation, associate with (Gibbings et al., 2009) and regulate (Lee et al., 2009) miRNA-induced silencing complexes (miRSC) support dysregulation of miRNA activity as a mechanism driving this aberrant resolution phenotype. Inhibition of MVB formation results in decreased miRSC activity, while blockage of MVB turnover leads to over-accumulation of miRSCs (Gibbings et al., 2009; Lee et al., 2009). Our study shows that Rab27a knockdown, which causes cytoplasmic accumulation of MVBs (Ostrowski et al., 2010), phenocopies the effects of blockage of MVB turnover on miRSC activity. It is also plausible that increased miR155 sorting into exosomes is caused by the decrease in pro-inflammatory genes targets during inflammatory resolution, based on a recent study showing that miRNAs against lowly expressed transcripts are directed towards exosomal secretion (Squadrito et al., 2014). Nonetheless, this scenario still supports our observations, as intracellular miR-155 accumulation would result in aberrant regulation of both lowly and highly expressed target transcripts during resolution. While our study focuses on miR-155, we do not exclude the role of other miRNAs and proteins given the heterogeneity in microglia exosome cargo and their associated biological functions. Indeed, gene ontol- 


\section{bioR Xiv}

ogy analyses of microglia exosome proteins suggests their involvement in protein trafficking and this is supported by a recent study showing that loss of Rab27a alters NADPH oxidase trafficking in activated macrophages and microglia (Ejlerskov et al., 2012). Further studies are necessary to better elucidate the diversity of exosome regulatory mechanisms and their relevance to different aspects of microglia inflammation.

Finally, we show dramatic alteration of exosome biogenesis in the aged brain, characterized by increased microglia exosome release and interstitial exosome abundance. Similar to our findings in acutely activated microglia, inhibition of exosome biogenesis in aged microglia exacerbates inflammatory activation. Transcriptional profiling recently revealed stronger anti-inflammatory gene profiles in aged compared to young microglia (Hickman et al., 2013). Our findings suggest exosome release as a possible effector of such anti-inflammatory gene networks in aged microglia. More work is, however, needed to understand interactions between exosomes and other microglia homeostatic mechanisms (Deczkowska et al., 2018; Matcovitch-Natan et al., 2016). Additionally, understanding the effects of dramatic endolysosomal reorganization in aged microglia on exosome cargo sorting and activity will better inform functional manipulation of exosomes in aged microglia. In summary, our findings identify exosome release as a novel anti-inflammatory effector mechanism utilized by acute and chronically activated microglia to restore immune homeostasis, and demonstrate a cell-state dependent dynamic interaction between exosomes, miRNAs and inflammation in microglia.

\section{MATERIALS AND METHODS}

Animal Models. The following mouse lines were used: C57BL/6 (The Jackson Laboratory), C57BL/6 aged mice (National Institutes of Aging), $\mathrm{C} 3 \mathrm{H} / \mathrm{HeSnJ}$ wildtype and ashen mutant mice (The Jackson Laboratory). All studies were done in male mice. The numbers of mice used to result in statistically significant differences was calculated using standard power calculations with $\alpha=0.05$ and a power of 0.8 . Power and size were calculated based on with the respective tests, variability of the assays and inter-individual differences within groups using http://www.stat.uiowa. edu/ rlenth/Power/index.html. Mice were housed under specific pathogen-free conditions under a $12 \mathrm{~h}$ light-dark cycle and all animal handling and use was in accordance with institutional guidelines approved by the University of California San Francisco IACUC.

Stereotaxic injections. Animals were placed in a stereotaxic frame and anesthetized with $2 \%$ isoflurane (2L/ min oxygen flow rate) delivered through an anesthesia nose cone. Ophthalmic eye ointment (Puralube Vet

\section{ARTICLE PREPRINT}

Ointment, Dechra) was applied to the cornea to prevent desiccation during surgery. The area around the incision was trimmed. Solutions (GW4869 or DMSO dissolved in PBS) were injected unilaterally into the DG of the dorsal hippocampi using the following coordinates: (from bregma) anterior $=-2 \mathrm{~mm}$, lateral $=$ $1.5 \mathrm{~mm}$, (from skull surface) height $=-2.1 \mathrm{~mm}$. A $2.5 \mu \mathrm{l}$ volume was injected stereotaxically over 10 minutes (injection speed: $0.20 \mu \mathrm{l} / \mathrm{min}$ ) using a $5 \mu \mathrm{l} 26 \mathrm{~s}$ gauge Hamilton syringe. To limit reflux along the injection track, the needle was maintained in situ for four minutes, slowly pulled out half way and kept in position for an additional two minutes. The skin was closed using silk suture. Each mouse was injected subcutaneously with the analgesic Buprenex. Mice were single-housed and monitored during recovery. Mice were perfused 7 days post-injection for tissue analyses.

Intracerebroventricular (ICV) delivery. Osmotic pumps were first attached to an L-shaped cannula via a $3.5 \mathrm{~cm}$ tubing according to manufacturer's instructions (Model 1002, Brain infusion kit 3). The assembly was preincubated in normal saline at $37 \mathrm{C}$ for $48-72 \mathrm{hrs}$ to activate pump prior to surgical implantation. The assembly was surgically implanted into the left ventricle of each mouse using a stereotactic apparatus, using the following coordinates: (from bregma) anterior $=-0.4 \mathrm{~mm}$, lateral $=1.0 \mathrm{~mm}$, (from skull surface) height $=-2.5 \mathrm{~mm}$. The osmotic pumps were place under the skin of the left flank by making a subcutaneous pocket with a curved, blunt scissors. Cannula were secured by gluing to the skull surface, prior to stitching the skin back together. Pumps contained either $10 \mathrm{ng} / \mathrm{ml}$ IFN $\gamma$ dissolved in PBS or just PBS. Pumps were loaded with a maximum volume of $100 \mu \mathrm{l}$ and pumped at a rate of $6 \mu \mathrm{l}$ per day. Pumps were implanted for either 3 or 7 days, before mice were sacrificed and brains harvested for histology, RNA and protein analyses.

\section{Microglia culture and isolation}

BV2 microglia culture. The BV2 microglia cell line was maintained in growth media - DMEM (Thermo Fisher) supplemented with $10 \%$ heat-inactivated fetal bovine serum (FBS, Hyclone) and $1 \%$ penicillin/streptomycin (Life Technologies) in HERAcell 150i incubators (Caisson Labs) at 37C with $5 \%$ CO2. BV2 microglia were serially passaged once plates reached $80-90 \%$ confluency.

\section{Postnatal primary microglia isolation and culture.} Primary microglial cells were harvested from mouse pups at postnatal day 3-6 (P3-P6). Briefly, the brain cortices were isolated and minced. Tissues were dissociated in $0.25 \%$ Trypsin-EDTA for $20 \mathrm{~min}$ at $37^{\circ} \mathrm{C}$ and agitated every $5 \mathrm{~min}$. Trypsin was neutralized with complete medium (DMEM (Thermo Fisher) supplemented with $10 \%$ heat-inactivated fetal bovine serum (FBS, Hyclone)), and were filtered through $70 \mu \mathrm{m}$ cell strainers (BD Falcon) and pelleted by centrifugation at $1500 \mathrm{rpm}$. Mixed glial cultures were maintained in growth medium at $37^{\circ} \mathrm{C}$ and $5 \%$ CO2 for $7-10$ d in vitro. Once bright round cells began to appear in the mixed 


\section{bioR $\chi$ iv}

glial cultures, recombinant mouse granulocyte macrophage colony stimulating factor $(1 \mathrm{ng} / \mathrm{ml}$, Life Technologies) was added to promote microglia proliferation. Primary microglial cells were harvested by mechanical agitation after 48-72 hours and plated on poly-L lysine coated t-75 flasks (Corning) in growth media and used for functional assays within 72 hours of purification. Functional assays were performed in serum free media.

Adult primary mouse microglia isolation. Wild type mice ( 3 and 24 months, National Institutes of Aging) were transcardially perfused with PBS, brains gently minced, and dissociated into single cells using the Neural Tissue Dissociation Kit P (Miltenyl Biotec). Cells were washed with Hank's Balanced Salt Solution and resuspended in PBS containing $0.5 \%$ FBS and $2 \mathrm{mM}$ EDTA (MACS buffer). Myelin removal was performed by incubation of the cell suspension with a ferric anti-myelin antibody and passed through a LS separation column placed in a magnetic holder (Miltenyl Biotec). Microglia enrichment was performed on the flow through cells by incubation with CD11b microbeads (Miltenyl Biotec), washed with MACS buffer and applied to a LS separation column placed in a magnetic holder (Miltenyl Biotec). The columns were removed from the magnetic field and labeled cells were flushed from the column with MACS buffer. Flow cytometry on the labeled cells confirmed $\sim 95 \%$ microglia purity. Isolated microglia were immediately processed for RNA isolation without culture or frozen in RNA stabilization buffer and frozen (RNAlater-ICE, Ambion). Quantitative PCR was performed on the samples using Taqman primers for IL $1 \beta$, TNF $\alpha$, IL10 and Gapdh (Life Technologies).

\section{Exosome isolation and characterization}

Isolation from microglia culture media. BV2 or primary microglia were grown to $\sim 60-70 \%$ confluency, thoroughly washed with PBS, and incubated in macrophage serum free media (MSFM) with or without interferon gamma. MSFM was collected after overnight incubation and processed for exosome isolation by differential ultracentrifugation, as previously described(Thery et al., 2006). Briefly, media was spun for $300 \mathrm{xg}$ for $10 \mathrm{~min}$ to pellet live cells. Dead cells and apoptotic bodies were removed by sequential spins of 2,000 x g and 10,000 $x \mathrm{~g}$, respectively. The supernatant was then passed through a $0.22 \mu \mathrm{m}$ syringe filter unit. Exosomes were pelleted finally pelleted by centrifuging at $100,000 \times \mathrm{g}$ for 90 mins. Pelleted exosomes were then washed with $30 \mathrm{ml}$ filtered PBS at $100,000 \times \mathrm{g}$ for 90 mins. Ultracentrifugation was done using SW 28 or Ti 70.1 rotors on a Beckmann Coulter Optima XPN-80 ultracentrifugation. For mass spectrometry and Taqman microRNA profiling, exosomes were further concentrated using total exosome isolation buffer (Life Technologies). Isolation of interstitial brain exosome: Mouse brains were processed for exosome isolation following published techniques(Perez-Gonzalez et al., 2012). Briefly, freshly perfused or frozen hemibrains were gently homogenized in dissociation buffer - Hibernate E (BrainBits Inc.) plus papain (Wormingthon) and incubated at $37 \mathrm{C}$ for 15 mins. Digestion was quenched by diluting

\section{ARTICLE PREPRINT}

in twice the volume of Hibernate E, filtered through a $40 \mathrm{um}$ mesh to eliminate debris. Further filtration was performed using a $0.22 \mu \mathrm{m}$ syringe filter. Differential ultracentrifugation was performed as described above to isolate exosomes. To concentrate exosomes into a smaller volume, the resuspended pellet from differential ultracentrifugation was incubated with total exosome isolation buffer (Life Technologies) overnight. Samples were centrifuged at 10000 $\mathrm{x} \mathrm{g}$ for 60 mins, resuspended in sterile PBS, and utilized for downstream applications or stored at $-80 \mathrm{C}$. Nanoparticle tracking. Size distribution of the purified exosomes was determined using nanoparticle tracking analysis (Malvern Inc.). To get consistent measurements on the nanoparticle tracking software, purified exosomes were diluted hundred-fold or more in cold, filtered PBS to obtain an ideal vesicle concentration range. 3-4 measurements of each sample were collected and concentration values were averaged. Transmission electron microscopy. Exosomes were fixed in EM grade formaldehyde immediately following isolation. Negative staining was performed on a small aliquot of the fixed exosomes, which were loaded on Formvar-carbon coated EM grids and negative staining was performed as previously described(Thery et al., 2006). Scanning electron microscopy allowed visualization of vesicle structure and size. Western Blot. Exosomes were lysed in RIPA buffer (500 mM Tris, pH 7.4, $150 \mathrm{mM} \mathrm{NaCl,} 0.5 \%$ sodium deoxycholate, $1 \%$ NP40, $0.1 \%$ SDS, and complete protease inhibitors; Roche) or exosome lysis buffer (Life Technologies). Lysates were stored at -80C or used immediately for protein analysis. Lysates were mixed with 4x NuPage LDS loading buffer (Invitrogen) and loaded on a $10 \%$ SDS polyacrylamide gradient gel (Invitrogen) and subsequently transferred onto a nitrocellulose membrane. The blots were blocked in 5\% milk in Tris-Buffered Saline with Tween (TBST) and incubated with rat anti-CD63 (1:500; MBL; clone: R5G2), rat anti-CD9 (1:2000, Biosciences; clone: KMC8), and rat anti-LAMP2 (1:200, Santa Cruz; clone: M3/84). CD9 and CD63 were run in non-reduced conditions. Protein lysates from BV2 microglia were probed with rabbit anti-phospho Stat1 (Tyr701, Cell Signaling), mouse anti-tubulin (Proteintech), and mouse anti-Gapdh (Sigma). Horseradish peroxidase-conjugated secondary antibodies (1:5000, GE Healthcare; NA934) and an ECL kit (Biorad) were used to detect protein signals. Multiple exposures were taken to select images with appropriate exposure (Biorad Imager). Selected images were exported at 300dpi and quantified using Image J software (Version 1.46k). Exosome Western blots were normalized to total protein concentration. Liquid chromatography mass Spectrometry. Pooled exosomes were lysed and denatured in an aqueous solution of $50 \mathrm{mM}$ TEAB and $0.1 \%$ SDS (Sigma), Protein concentration was measured using a BCA assay, and $25-50 \mu \mathrm{g}$ of each sample placed in a speedvac at $60 \mathrm{C}$. Samples were reduced in $500 \mathrm{mM}$ TECP for 1 hour at $60 \mathrm{C}$ and alkylated by addition of $10 \mathrm{mM}$ iodoacetamide for 15 mins at room temperature. Tryp- 


\section{bioR $\chi$ iv}

sin (spectrometry grade, Promega) was added to the solution at ratio of 1:25 and incubate overnight at 37C. Detergent removal was performed on the samples following manufacturer's instructions (Thermo Fisher). Samples placed in a speedvac until nearly dry and resuspended in to a concentration of $1 \mu \mathrm{g} / \mu \mathrm{l}$ in LCMS buffer $A$ and stored at $-80 \mathrm{C}$. Peptides were separated using a nanoLC Ultra 2D Plus CHIPLC system (SClEX) in serial two column mode with two nano cHIPLC columns (75 $\mu \mathrm{m} \times 15 \mathrm{~cm}$ ChromXP C18-CL $3 \mu \mathrm{m} 300$ $\AA$ ). The peptides were initially loaded onto the first column and washed with buffer A (2\% acetonitrile/98\% $\mathrm{H} 2 \mathrm{O} / 0.1 \%$ formic acid) for $30 \mathrm{~min}$ at a flow rate of 0.5 $\mu \mathrm{L} / \mathrm{min}$. The elution gradient was $2-30 \%$ buffer B $(98 \%$ acetonitrile/2\% $\mathrm{H} 2 \mathrm{O} / 0.1 \%$ formic acid) over $120 \mathrm{~min}$ at $300 \mathrm{~nL} / \mathrm{min}$. The TripleTOF 5600 equipped with a NanoSpray III source (SCIEX) was used for MS data acquisition. The IDA method was constructed to acquire a TOF MS survey scan at $>30,000$ resolution for 0.25 msec, followed by $20 \mathrm{MS} / \mathrm{MS}$ spectra in $3 \mathrm{~s}$ at $>15,000$ resolution with an exclusion time of $15 \mathrm{~s}$. Protein identification was performed by using ProteinPilot v5.0 software (SCIEX) and the UniProt SwissProt v2011605 Mus musculus database using integrated false discovery rate analysis function with a concatenated reversed database. Data were searched in thorough mode with tryspin digestion and iodoacetamide modification. Proteins detected with local false discovery rate (FDR) $\leq 5 \%$ from each individual experiment were aligned to a search result from all samples searched together (Master) to create a comprehensive list of proteins and compare groups. The Master list for alignment included191 proteins detected with a global FDR $\leq 1 \%$. From the Master list, only proteins detected in replicates within each group were considered for final comparisons. microRNA profiling. Exosomes were pooled from several experiments to have sufficient input material. Pooled samples of BV2 microglia exosomes lysed and processed for microRNA isolation using the miRNeasy kit according to manufacturer's instructions (Qiagen). To measure miRNA composition, reverse transcription and preamplification was performed using Rodent miRNA primer pool $A$ and $B$ on 300ng of input RNA isolated from pooled activated and unactivated BV2 exosomes (Life Technologies). To measure the abundance of individual miRNA species, samples were loaded into Taqman Rodent Array microRNA A and B cards (version 3.0, Applied Biosystems) and quantitative PCR was performed. Resultant Ct values for each sample were normalized to U6 levels, and fold change was determined relative to untreated exosome miRNAs. miRNA expression in interferon gamma (IFN $\gamma$, Life Technologies) treated microglia was performed using taqman miRNA assays (Life Technologies) against mmu-miR-155, has-miR-125-5p, has-miR-103, snU6. Following reverse transcription, real time PCR was performed to measure expression of miRNAs across different samples. Expression was normalized to U6 levels.

Immunohistochemistry. Tissue processing and immunohistochemistry was performed on free-floating sec-

\section{ARTICLE PREPRINT}

tions following standard published techniques(Villeda et al., 2011). Briefly, mice were anesthetized with $400 \mathrm{mg} / \mathrm{kg}$ avertin (Sigma-Aldrich) and transcardially perfused with $0.9 \%$ saline. Brains were removed and fixed in phosphate-buffered $4 \%$ paraformaldehyde, $\mathrm{pH} \mathrm{7.4,} \mathrm{at} \mathrm{4C} \mathrm{for} 48 \mathrm{~h}$ before they were sunk through $30 \%$ sucrose for cryoprotection. Brains were then sectioned coronally at $40 \mu \mathrm{m}$ with a cryomicrotome (Zeiss, Inc.) and stored in cryoprotective medium. Primary antibodies were: rabbit anti-Iba1 (1:2000, Dako), rat anti-CD63 (1:100, Santa Cruz) and rat anti-CD68 (1:200, Millipore). After overnight incubation, primary antibody staining was revealed using fluorescence conjugated secondary antibodies (Life Technologies).

Viral Infection. BV2 microglia were maintained in DMEM media containing 10\% FBS. Cells were transduced with lentivirus (LV) containing an shRNA plasmid targeting mouse rab27a or a scramble shRNA sequence using a previously published transduction protocol(Lucin et al., 2013). The lentivirus also expressed a copped GFP, to allow visualization of infected cells. BV2 were plated at a density of 1000 cells per well in 96-well plates, and infected at a multiplicity of infection of 50 or 100 in the presence of polybrene $(8 \mathrm{mg} / \mathrm{ml})$. After overnight infection, media was removed and replaced with normal growth media. Cells were allowed to grow for an additional 72 hours, split into 24 well dishes and treated with puromycin to select for infected cells. Selection was performed for 48 hours and surviving cells were assessed for GFP expression using the Accuri C6 machine. Knockdown was confirmed qPCR assessment of rab27a transcript levels, due to lack of a good commercial antibody. LV plasmids were prepared by performing lipofectamine transfection of 293T cells with vectors expressing desired transgene, Pax2, and VSVG. Following overnight incubation, transfection media was replaced with viral production media, DMEM plus viral boost reagent (Alstem). Virus production media was collected the following day, centrifuged to pellet cells and debris, and filtered through a 0.45 mesh (Maine Productions). Virus containing media was concentrated by high speed ultracentriguation, resuspended in PBS, aliquoted, and used immediately for experiments or stored at $-80 \mathrm{C}$. Data and statistical analyses. All experiments were randomized and blinded by an independent researcher prior to pharmacological treatment or assessment of genetic mouse models. Researchers remained blinded throughout histological and biochemical assessments. Groups were un-blinded at the end of each experiment upon statistical analysis. Data are expressed as mean \pm SEM. The distribution of data in each set of experiments was tested for normality using D'Agostino-Pearson omnibus test or Shapiro-Wilk test. No significant differences in variance between groups were detected using an $F$ test. Statistical analysis was performed with Prism 5.0 software (GraphPad Software). Means between two groups were compared with two-tailed Student's t test. Comparisons of means from multiple groups with each 


\section{bioR $\chi$ iv}

other or against one control group were analyzed with 1-way ANOVA followed by appropriate post-hoc tests.

\section{REFERENCES}

Baruch, K., Deczkowska, A., David, E., Castellano, J.M., Miller, O., Kertser, A., Berkutzki, T., Barnett-Itzhaki, Z., Bezalel, D., Wyss-Coray, T., et al. (2014). Aging. Aging-induced type I interferon response at the choroid plexus negatively affects brain function. Science 346, 89-93.

Butovsky, O., Jedrychowski, M.P., Cialic, R., Krasemann, S., Murugaiyan, G., Fanek, Z., Greco, D.J., Wu, P.M., Doykan, C.E., Kiner, O., et al. (2015). Targeting miR-155 restores abnormal microglia and attenuates disease in SOD1 mice. Ann Neurol 77, 75-99.

Deczkowska, A., Amit, I., and Schwartz, M. (2018). Microglial immune checkpoint mechanisms. Nat Neurosci 21, 779-786.

Ejlerskov, P., Christensen, D.P., Beyaie, D., Burritt, J.B., Paclet, M.H., Gorlach, A., van Deurs, B., and Vilhardt, F. (2012). NADPH oxidase is internalized by clathrin-coated pits and localizes to a Rab27A/B GTPase-regulated secretory compartment in activated macrophages. J Biol Chem 287, 4835-4852.

Fitzner, D., Schnaars, M., van Rossum, D., Krishnamoorthy, G., Dibaj, P., Bakhti, M., Regen, T., Hanisch, U.K., and Simons, M. (2011). Selective transfer of exosomes from oligodendrocytes to microglia by macropinocytosis. J Cell Sci 124, 447-458.

Fruhbeis, C., Frohlich, D., Kuo, W.P., Amphornrat, J., Thilemann, S., Saab, A.S., Kirchhoff, F., Mobius, W., Goebbels, S., Nave, K.A., et al. (2013). Neurotransmitter-triggered transfer of exosomes mediates oligodendrocyte-neuron communication. PLoS Biol 11, e1001604.

Gibbings, D.J., Ciaudo, C., Erhardt, M., and Voinnet, O. (2009). Multivesicular bodies associate with components of miRNA effector complexes and modulate miRNA activity. Nat Cell Biol 11, 1143-1149.

Grabert, K., Michoel, T., Karavolos, M.H., Clohisey, S., Baillie, J.K., Stevens, M.P., Freeman, T.C., Summers, K.M., and McColl, B.W. (2016). Microglial brain region-dependent diversity and selective regional sensitivities to aging. Nat Neurosci 19, 504-516.

Hickman, S.E., Kingery, N.D., Ohsumi, T.K., Borowsky, M.L., Wang, L.C., Means, T.K., and El Khoury, J. (2013). The microglial sensome revealed by direct RNA sequencing. Nat Neurosci 16, 1896-1905. Jablonski, K.A., Gaudet, A.D., Amici, S.A., Popovich, P.G., and Guerau-de-Arellano, M. (2016). Control of
ARTICLE PREPRINT

the Inflammatory Macrophage Transcriptional Signature by miR-155. PLoS One 11, e0159724.

Kettenmann, H., Hanisch, U.K., Noda, M., and Verkhratsky, A. (2011). Physiology of microglia. Physiol Rev 91, 461-553.

Klumperman, J., and Raposo, G. (2014). The complex ultrastructure of the endolysosomal system. Cold Spring Harb Perspect Biol 6, a016857.

Kowal, J., Arras, G., Colombo, M., Jouve, M., Morath, J.P., Primdal-Bengtson, B., Dingli, F., Loew, D., Tkach, M., and Thery, C. (2016). Proteomic comparison defines novel markers to characterize heterogeneous populations of extracellular vesicle subtypes. Proc Natl Acad Sci U S A 113, E968-977.

Lee, Y.S., Pressman, S., Andress, A.P., Kim, K., White, J.L., Cassidy, J.J., Li, X., Lubell, K., Lim, D.H., Cho, I.S., et al. (2009). Silencing by small RNAs is linked to endosomal trafficking. Nat Cell Biol 11, 1150-1156.

Lindenbergh, M.F.S., and Stoorvogel, W. (2018). Antigen Presentation by Extracellular Vesicles from Professional Antigen-Presenting Cells. Annu Rev Immunol 36, 435-459.

Lucin, K.M., O’Brien, C.E., Bieri, G., Czirr, E., Mosher, K.I., Abbey, R.J., Mastroeni, D.F., Rogers, J., Spencer, B., Masliah, E., et al. (2013). Microglial beclin 1 regulates retromer trafficking and phagocytosis and is impaired in Alzheimer's disease. Neuron 79, 873-886.

Matcovitch-Natan, O., Winter, D.R., Giladi, A., Vargas Aguilar, S., Spinrad, A., Sarrazin, S., Ben-Yehuda, H., David, E., Zelada Gonzalez, F., Perrin, P., et al. (2016). Microglia development follows a stepwise program to regulate brain homeostasis. Science 353, aad8670.

Menasche, G., Pastural, E., Feldmann, J., Certain, S., Ersoy, F., Dupuis, S., Wulffraat, N., Bianchi, D., Fischer, A., Le Deist, F., et al. (2000). Mutations in RAB27A cause Griscelli syndrome associated with haemophagocytic syndrome. Nat Genet 25, 173-176. Mosher, K.I., and Wyss-Coray, T. (2014). Microglial dysfunction in brain aging and Alzheimer's disease. Biochem Pharmacol 88, 594-604.

Nimmerjahn, A., Kirchhoff, F., and Helmchen, F. (2005). Resting microglial cells are highly dynamic surveillants of brain parenchyma in vivo. Science 308, 1314-1318.

Ostrowski, M., Carmo, N.B., Krumeich, S., Fanget, I., Raposo, G., Savina, A., Moita, C.F., Schauer, K., Hume, A.N., Freitas, R.P., et al. (2010). Rab27a and Rab27b control different steps of the exosome secre- 


\section{bioRxiv}

tion pathway. Nat Cell Biol 12, 19-30; sup pp 11-13. Perez-Gonzalez, R., Gauthier, S.A., Kumar, A., and Levy, E. (2012). The exosome secretory pathway transports amyloid precursor protein carboxyl-terminal fragments from the cell into the brain extracellular space. J Biol Chem 287, 43108-43115.

Ponomarev, E.D., Veremeyko, T., and Weiner, H.L. (2013). MicroRNAs are universal regulators of differentiation, activation, and polarization of microglia and macrophages in normal and diseased CNS. Glia 61, 91-103.

Potolicchio, I., Carven, G.J., Xu, X., Stipp, C., Riese, R.J., Stern, L.J., and Santambrogio, L. (2005). Proteomic analysis of microglia-derived exosomes: metabolic role of the aminopeptidase CD13 in neuropeptide catabolism. J Immunol 175, 2237-2243.

Prinz, M., Priller, J., Sisodia, S.S., and Ransohoff, R.M. (2011). Heterogeneity of CNS myeloid cells and their roles in neurodegeneration. Nat Neurosci 14, 1227-1235.

Robbins, P.D., and Morelli, A.E. (2014). Regulation of immune responses by extracellular vesicles. Nat Rev Immunol 14, 195-208.

Sarper, N., Akansel, G., Aydogan, M., Gedikbasi, D., Babaoglu, K., and Gokalp, A.S. (2002). Neuroimaging abnormalities in Griscelli's disease. Pediatr Radiol 32, 875-878.

Sierra, A., Gottfried-Blackmore, A.C., McEwen, B.S., and Bulloch, K. (2007). Microglia derived from aging mice exhibit an altered inflammatory profile. Glia 55, 412-424.

Squadrito, M.L., Baer, C., Burdet, F., Maderna, C., Gilfillan, G.D., Lyle, R., Ibberson, M., and De Palma, M. (2014). Endogenous RNAs modulate microRNA sorting to exosomes and transfer to acceptor cells. Cell Rep 8, 1432-1446.

Thery, C., Amigorena, S., Raposo, G., and Clayton, A. (2006). Isolation and characterization of exosomes from cell culture supernatants and biological fluids. Curr Protoc Cell Biol Chapter 3, Unit 322.

Trajkovic, K., Hsu, C., Chiantia, S., Rajendran, L., Wenzel, D., Wieland, F., Schwille, P., Brugger, B., and Simons, M. (2008). Ceramide triggers budding of exosome vesicles into multivesicular endosomes. Science (New York, NY) 319, 1244-1247.

Udeochu, J.C., Shea, J.M., and Villeda, S.A. (2016).

Microglia communication: Parallels between aging and Alzheimer's disease. Clin Exp Neuroimmunol 7, 114125.

Villeda, S.A., Luo, J., Mosher, K.I., Zou, B., Britschgi, M., Bieri, G., Stan, T.M., Fainberg, N., Ding, Z., Eggel,

\section{ARTICLE PREPRINT}

A., et al. (2011). The ageing systemic milieu negatively regulates neurogenesis and cognitive function. Nature 477, 90-94.

Wang, P., Hou, J., Lin, L., Wang, C., Liu, X., Li, D., Ma, F., Wang, Z., and Cao, X. (2010). Inducible microRNA-155 feedback promotes type I IFN signaling in antiviral innate immunity by targeting suppressor of cytokine signaling 1. J Immunol 185, 6226-6233. Wong, W.T. (2013). Microglial aging in the healthy CNS: phenotypes, drivers, and rejuvenation. Front Cell Neurosci 7, 22.

Ye, S.M., and Johnson, R.W. (1999). Increased interleukin- 6 expression by microglia from brain of aged mice. J Neuroimmunol 93, 139-148.

\section{ACKNOWLEDGEMENTS}

This work was funded by NSF predoctoral fellowship (J.C.U), Society for Neuroscience Scholars Program (J.C.U), Glenn Foundation (S.A.V), NIA (R01 AG053382, R01 AG055797).

Supplemental material upload as separate pdf 